\title{
Observer-based leader-following formation control of uncertain multiple agents by integral sliding mode
}

\author{
D.W. QIAN ${ }^{1 *}$, S.W. TONG ${ }^{2}$, and C.D. LI $^{3}$ \\ ${ }^{1}$ School of Control and Computer Engineering, North China Electric Power University, Beijing, 102206 China \\ ${ }^{2}$ College of Automation, Beijing Union University, Beijing, 100101 China \\ ${ }^{3}$ School of Information and Electrical Engineering, Shandong Jianzhu University, Jinan, 250101 China
}

\begin{abstract}
This paper investigates the formation control problem of multiple agents. The formation control is founded on leader-following approaches. The method of integral sliding mode control is adopted to achieve formation maneuvers of the agents based on the concept of graph theory. Since the agents are subject to uncertainties, the uncertainties also challenge the formation-control design. Under a mild assumption that the uncertainties have an unknown bound, the technique of nonlinear disturbance observer is utilized to tackle the issue. According to a given communication topology, formation stability conditions are investigated by the observer-based integral sliding mode formation control. From the perspective of Lyapunov, not only is the formation stability guaranteed, but the desired formation of the agents is also realized. Finally, some simulation results are presented to show the feasibility and validity of the proposed control scheme through a multi-agent platform.
\end{abstract}

Key words: multi-agent system, formation control, integral sliding mode control, uncertainty, nonlinear disturbance observer, stability.

\section{Introduction}

Recently, multi-agent systems have been paid considerable attention [1-3]. The technology of multi-agent systems has been praised as a novel paradigm for designing and implementing many real systems, such as distributed sensor networks [4], robots [5-8], satellite flying [9] and aerial vehicles [10]. In reality, a multi-agent system has some advantages over a single agent, including but not limited to efficiency, reliability, extensibility, robustness and flexibility $[11,12]$. Increasing applications involve multiple agents that have to work together, which implies the requirement of multiple agents coordination.

Among a variety of cooperative tasks, the consensus problem becomes attractive because it integrates both graph theory and control theory. The consensus problem contains several typical control problems, i.e., cooperative control, formation control and flocking. See [13] for a complete review of recent approaches to this area. As a branch, formation control works on controlling agents in a multi-agent system to form up and move in specified geometrical shapes [14]. The background of formation control originates from the real world. For instance, the agents need to maintain some formations when they move at disaster sites, warehouses and hazardous areas.

One of coordinated control schemes for multi-agent formations is defined as leader-following; one agent is designed as the leader and other agents are followers. The sole leader is self-commanded and moves along a predefined trajectory. The followers track the leader. A cooperative task can be achieved by the interactions between these followers and the leader. The scheme in $[4,6,9,10]$ has been successfully applied to the anal-

*e-mail: dianwei.qian@ncepu.edu.cn ysis and design of multiple agent systems. However, the classic leader-following scheme is centralized, meaning that the control systems in $[4,6,9,10]$ heavily depend on the leader and suffer from the "single point of failure" problem. With the development of communication technology, it is desired to consider the communication topology in the scheme because the adaptability and practicability of multiple agents can be strengthened $[15,16]$.

The leader-following scheme gains popularity in multi-agent formations because its dynamics are experimentally modelled, but the internal formation stability can be theoretically guaranteed. Adopting such a scheme, various control methods have been developed for multi-agent formations, that is, robust control [17], dynamic output feedback method [18], adaptive fuzzy approach [19], multi-step predictive mechanism [20], iterative learning technique [21], and neural network-based adaptive design [22], to name but a few. A systematic review on this topic is presented by Oh, Park and Ahn [23].

As a nonlinear feedback design tool, the sliding mode control (SMC) technique is popular because of its invariance [24], meaning that matched uncertainties in an SMC system can be suppressed by the invariance. Some SMC-based methods have been addressed to solve the formation-control problem of multi-agent systems, that is, first-order SMC $[25,26]$, terminal SMC [27], backstepping SMC [28], etc. Previous contributions $[15,25-28]$ have verified the feasibility of the SMC methodology for multi-agent formations.

Compared to other SMC methods, the integral SMC design can guarantee an integral SMC system against matched uncertainties from the initial time, indicating that an entire response of the system is of invariance. Some successful applications of the integral SMC method have been reported in industries, for example, power systems [29], hypersonic vehicles [30] as well as multi-agent systems $[15,31,32]$. 
Uncertainties exist everywhere. Each individual agent may suffer from uncertainties, i.e., external disturbances, unmodelled dynamics and parameter perturbations. Originated from the uncertainties of individual agents, formation dynamics of multiagent systems become uncertain. In previous works on the SMCbased multi-agent formations [15, 25-28, 31, 32], uncertainties are considered because they adversely affect the formation stability. Two solutions can be summarized from those works. One solution is to discuss the formation stability by means of graph theory $[15,32]$. The other is to analyze the formation stability in light of Lyapunov's theorem [25-28, 31]. To guarantee the formation stability, both solutions have to assume that the formation uncertainties are bounded. Unfortunately, the boundary of uncertainties is rather hard to exactly measure or know in advance. The lack of such a boundary may result in severe problems, i.e., decrease of the formation robustness, deterioration of the formation performance as far as deficiency of the formation stability. To obtain the important information, adaptive approximation of the formation uncertainties is desired.

The technique of nonlinear disturbance observer (NDO) has been proven to be effective in handling uncertainties and improving robustness [33]. The applications of NDO have been investigated in several cases $[34,35]$. Such a technique can be considered as an alternative solution to the problem of uncertainties. So far, the academic problem of how to eliminate the adverse effects of uncertainties in multi-agent formations via NDO still remains unsolved and problematic.

This paper addresses the academic problem. An NDO-based observer is employed to observe the uncertainties of agents. Based on the observer, an integral sliding mode controller is developed to achieve the formation maneuvers of agents. The formation stability is presented in Lyapunov sense. By comparison, numerical results demonstrate that the presented control design is preferable.

\section{Modelling}

2.1. One single agent. A multi-agent system contains $N$ identical agents. Displayed in Fig. 1, the single agent under consideration is a differential wheeled robot in the horizon. The robot

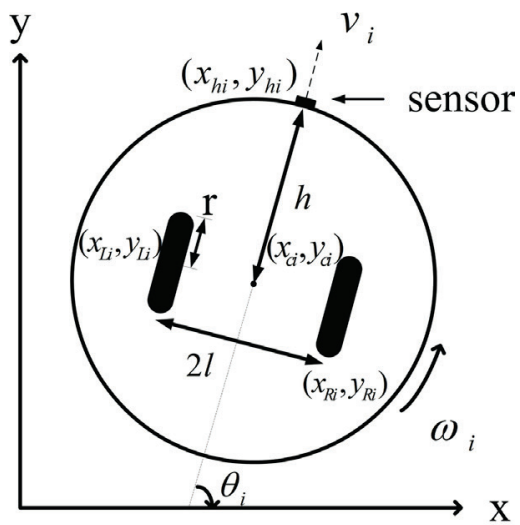

Fig. 1. Schematic diagram of a single agent is round with a radius of $r$. Use index $i$ to refer to the robot in Fig. 1. The robot's movement is based on two separately driven wheels placed on either side of the robot's body, where $\left(x_{c i}, y_{c i}\right)$ are the center of this robot, $\left(x_{L i}, y_{L i}\right)$ are the center of the left wheel, $\left(x_{R i}, y_{R i}\right)$ are the center of the right wheel and are the center of the robot's body. Then, a vector $\mathbf{q}_{i}=\left[x_{h i} y_{h i} \theta_{i}\right]^{T}$ can be defined to describe the robot's posture, where $\left(x_{h i}, y_{h i}\right)$ are the robot's head in the inertial frame and $\theta_{i}$ denotes the orientation angle of a mobile frame [15]. Under the assumption of pure rolling and non-slipping motion, the robot is subjected to $\left[-\sin \theta_{i} \cos \theta_{i} 0\right] \cdot\left[x_{c i} y_{c i} \theta_{i}\right]=0$.

The Lagrangian equation of the agent can be obtained as $L_{i}=K_{i}-P_{i}$, where $K_{i}$ denotes the kinetic energy of the agent and $P_{i}$ means the potential energy. Concerning the agent, it can only move in the horizon so that its potential energy remains unchanged, that is, $P_{i} \equiv 0$. Then, $L_{i}$ can be written by

$$
L_{i}=K_{b i}+K_{l i}+K_{r i}
$$

In (1), $K_{b i}=\frac{1}{2} m_{b}\left(\dot{x}_{c i}^{2}+\dot{y}_{c i}^{2}\right)+\frac{1}{2} I_{b} \dot{\theta}_{i}^{2}, K_{l i}=\frac{1}{2} m_{w}\left(\dot{x}_{l i}^{2}+\dot{y}_{l i}^{2}\right)+$ $+\frac{1}{2} I_{w} \dot{\theta}_{i}^{2}$ and $K_{r i}=\frac{1}{2} m_{w}\left(\dot{x}_{r i}^{2}+\dot{y}_{r i}^{2}\right)+\frac{1}{2} I_{w} \dot{\theta}_{i}^{2}$, where $m_{b}$ and $I_{b}$ are the mass and the moment of inertia of the robot's body, respectively; $m_{w}$ and $I_{b}$ are the mass and the moment of inertia of the robot's wheel, respectively.

The Lagrangian equations of motion can be formulated as (2) with respect to vector $\mathbf{q}_{i}$.

$$
\frac{\mathrm{d}}{\mathrm{d} t}\left(\frac{\partial L_{i}}{\partial \dot{\mathbf{q}}_{i}}\right)-\frac{\partial L_{i}}{\partial \mathbf{q}_{i}}=\mathbf{B}\left(\mathbf{q}_{i}\right) \tau_{i}
$$

where $\tau_{i}=\left[\tau_{L i} \tau_{R i}\right]^{T}$ is the torque vector applied to the wheels and $\mathbf{B}\left(\mathbf{q}_{i}\right)$ is a time-varying matrix. By the Lagrangian method, the dynamic model of the agent [15] can be formulated by

$$
\mathbf{M}\left(\mathbf{q}_{i}\right) \ddot{\mathbf{q}}_{i}+\mathbf{C}\left(\mathbf{q}_{i}, \dot{\mathbf{q}}_{i}\right) \dot{\mathbf{q}}_{i}=\mathbf{B}\left(\mathbf{q}_{i}\right) \tau_{i}
$$

where the matrices $\mathbf{M}\left(\mathbf{q}_{i}\right), \mathbf{C}\left(\mathbf{q}_{i}, \dot{\mathbf{q}}_{i}\right)$ and $\mathbf{B}^{T}\left(\mathbf{q}_{i}\right)$ in order are determined by $\left[\begin{array}{ccc}m & 0 & m h \sin \theta_{i} \\ 0 & m & -m h \cos \theta_{i} \\ m h \sin \theta_{i} & -m h \cos \theta_{i} & I\end{array}\right]$, $\left[\begin{array}{ccc}0 & 0 & m h \dot{\theta}_{i} \cos \theta_{i} \\ 0 & 0 & -m h \dot{\theta}_{i} \sin \theta_{i} \\ 0 & 0 & 0\end{array}\right]$ and $\frac{1}{r}\left[\begin{array}{ccc}\cos \theta_{i} & \sin \theta_{i} & -1 \\ \cos \theta_{i} & \sin \theta_{i} & 1\end{array}\right]$.

In (3), $I=I_{b}+2 I_{w}+2 m_{w}+2 m_{w} l^{2}+m_{b} h^{2}, m=m_{b}+2 m_{w}$, $h$ is the length between the agent's center and the head and $l$ is the distance between the agent's center and one wheel.

From Fig. 1, two symbols of the agent are unexplained, that is, the linear velocity $v_{i}$ and the rotation angular velocity $\omega_{i}$. Define a vector $\xi_{i}=\left[v_{i} \omega_{i}\right]^{T}$. Then, $\dot{\mathbf{q}}_{i}=T\left(\mathbf{q}_{i}\right) \xi_{i}$ exists, here $\mathbf{T}^{T}\left(\mathbf{q}_{i}\right)=\left[\begin{array}{ccc}\cos \theta_{i} & \sin \theta_{i} & 0 \\ -h \sin \theta_{i} & h \cos \theta_{i} & 1\end{array}\right]$. Substituting $\dot{\mathbf{q}}_{i}=T\left(\mathbf{q}_{i}\right) \xi_{i}$ into (3) yields 


$$
\tilde{\mathbf{M}}\left(\mathbf{q}_{i}\right) \dot{\xi}_{i}+\tilde{\mathbf{C}}\left(\mathbf{q}_{i}, \dot{\mathbf{q}}_{i}\right) \xi_{i}=\tilde{\mathbf{B}}\left(\mathbf{q}_{i}\right) \tau_{i}
$$

where $\mathbf{M}\left(\mathbf{q}_{i}\right), \tilde{\mathbf{C}}\left(\mathbf{q}_{i}, \dot{\mathbf{q}}_{i}\right)$ and $\tilde{\mathbf{B}}\left(\mathbf{q}_{i}\right)$ in order are determined by $\mathbf{T}^{T}\left(\mathbf{q}_{i}\right) \mathbf{M}\left(\mathbf{q}_{i}\right) \mathbf{T}\left(\mathbf{q}_{i}\right), \mathbf{T}^{T}\left(\mathbf{q}_{i}\right)\left[\mathbf{M}\left(\mathbf{q}_{i}\right) \dot{\mathbf{T}}\left(\mathbf{q}_{i}\right)+\mathbf{C}\left(\mathbf{q}_{i}, \dot{\mathbf{q}}_{i}\right) \mathbf{T}\left(\mathbf{q}_{i}\right)\right]$ and $\mathbf{T}^{T}\left(\mathbf{q}_{i}\right) \mathbf{B}\left(\mathbf{q}_{i}\right)=\frac{1}{r}\left[\begin{array}{cc}1 & 1 \\ -1 & 1\end{array}\right]$.

In (4), $\tilde{\mathbf{C}}\left(\mathbf{q}_{i}, \dot{\mathbf{q}}_{i}\right)=\mathbf{0}_{2 \times 2}$ where $\mathbf{0}_{2 \times 2}$ is a $2 \times 2$ zero matrix. The fact indicates that $\tilde{\mathbf{M}}\left(\mathbf{q}_{i}\right) \dot{\xi}=\tilde{\mathbf{B}}\left(\mathbf{q}_{i}\right) \tau_{i}$ can be deduced from (4). Provided $\operatorname{det}\left[\mathbf{M}\left(\mathbf{q}_{i}\right)\right] \neq 0$, the assumption means $\tilde{\mathbf{M}}^{-1}\left(\mathbf{q}_{i}\right)$ is invertible such that the the equations of motion describing the behaviour of the agent can be formulated by

$$
\dot{\xi}_{i}=\tilde{\mathbf{M}}^{-1}\left(\mathbf{q}_{i}\right) \tilde{\mathbf{B}}\left(\mathbf{q}_{i}\right) \tau_{i}
$$

Reconsider $\dot{\mathbf{q}}_{i}=T\left(\mathbf{q}_{i}\right) \xi_{i}$ such that the equations of motion of the agent at its head has a form of

$$
\left[\dot{x}_{h i} \dot{y}_{h i}\right]^{T}=\mathbf{H}\left[v_{i} \omega_{i}\right]^{T}
$$

where $\mathbf{H}=\left[\begin{array}{cc}\cos \theta_{i} & -h \sin \theta_{i} \\ \sin \theta_{i} & h \cos \theta\end{array}\right]$.

Differentiating (6) with respect to time $t$ yields

$$
\left[\begin{array}{c}
\ddot{x}_{h i} \\
\ddot{y}_{h i}
\end{array}\right]=\left[\begin{array}{c}
\dot{v}_{x i} \\
\dot{v}_{y i}
\end{array}\right]=\mathbf{H}^{-1}\left(\mathbf{q}_{i}\right) \tilde{\mathbf{B}}\left(\mathbf{q}_{i}\right) \tau_{i}+\left[\begin{array}{c}
\rho_{1 i} \\
\rho_{2 i}
\end{array}\right]
$$

where $\rho_{1 i}=-v_{i} \omega_{i} \sin \theta_{i}-h \omega_{i}^{2} \cos \theta_{i}$ and $\rho_{2 i}=-v_{i} \omega_{i} \cos \theta_{i}-$ $-h \omega_{i}^{2} \sin \theta_{i}$.

Define the control actions $\left[u_{x i} u_{y i}\right]^{T}=\mathbf{H} \tilde{\mathbf{M}}^{-1}\left(\mathbf{q}_{i}\right) \tilde{\mathbf{B}}\left(\mathbf{q}_{i}\right) \tau_{i}+$ $+\left[\rho_{1 i} \rho_{2 i}\right]^{T}$. (7) can have the form of

$$
\left[\begin{array}{c}
\ddot{x}_{h i} \\
\ddot{y}_{h i}
\end{array}\right]=\left[\begin{array}{c}
\dot{v}_{x i} \\
\dot{v}_{y i}
\end{array}\right]=\left[\begin{array}{c}
u_{x i} \\
u_{y i}
\end{array}\right]
$$

Consider the agent's uncertainties, Finally, the agent can be described by

$$
\left[\begin{array}{c}
\dot{x}_{h i} \\
\dot{v}_{x i} \\
\dot{y}_{h i} \\
\dot{v}_{y i}
\end{array}\right]=\left[\begin{array}{c}
v_{x i} \\
u_{x i}+\delta_{x i} \\
v_{y i} \\
u_{y i}+\delta_{y i}
\end{array}\right]
$$

where $\delta_{x i}$ and $\delta_{y i}$ are the uncertain terms.

Assumption 1: $\left\|\delta_{x i}\right\| \leq \delta_{x i}^{*}$ and $\left\|\delta_{y i}\right\| \leq \delta_{y i}^{*}$, where $\delta_{x i}^{*}>0$ and $\delta_{y i}^{*}>0$ are constant but unknown.

Assumption 2: Both $\delta_{x i}$ and $\delta_{y i}$ are slowly time-varying, indicating $\dot{\delta}_{x i} \simeq 0$ and $\dot{\delta}_{y i} \simeq 0$.

Assumption 1 indicates the uncertainties are bounded by an unknown constant, which is mild. From Assumption 2, $\delta_{x i}$ and $\delta_{y i}$ seem almost constant. Considering the technical contents, Assumption 2 indicates that the designed observer could evaluate or calculate $\delta_{x i}$ and $\delta_{y i}$ much faster than the change rates of $\delta_{x i}$ and $\delta_{y i}$. In this sense, $\delta_{x i}$ and $\delta_{y i}$ could be treated as almost constant by the observer.

2.2. Communication topologies. Re-consider the multi-agent system with $N$ agents. The communication topologies of such a system can be modelled using the theory of algebraic graph $[15,32]$. Define a directed graph $G=(V, E)$ composed of a vertex set $V$ and an edge set $E$, where $V=\left\{v_{1}, v_{2}, \cdots, v_{N}\right\}$, $E \subseteq V \times V$, the node $v_{i}$ denotes the $i$ th agent and $i=1,2, \cdots, N$. This paper investigates the directed graph in the multi-agent system. Consider the $i$ th agent whose collection of neighbors is defined as $N_{i}=\left\{v_{j} \in V:\left(v_{i}, v_{j}\right) \in E\right\}$. The ordered pair $\left(v_{i}, v_{j}\right) \in E$ means that the $j$ th agent can send information to the $i$ th agent, but not vice versa.

The weighted adjacency matrix $A$ of $G$ has a form of

$$
A=\left[\begin{array}{cccc}
a_{11} & a_{12} & \cdots & a_{1 N} \\
a_{21} & a_{22} & \cdots & a_{2 N} \\
\vdots & \vdots & \ddots & \vdots \\
a_{N 1} & a_{N 2} & \cdots & a_{N N}
\end{array}\right] \in R^{N \times N}
$$

where $a_{i j}$ indicates the weight of the pair $\left(v_{i}, v_{j}\right) ; \forall\left(v_{i}, v_{j}\right) \in E$, $\exists a_{i j}=1 ; \forall\left(v_{i}, v_{j}\right) \notin E, \exists a_{i j}=0$ and $a_{i i}=0$.

The degree matrix of $G$ is a diagonal matrix, determined by $D=\operatorname{diag}\left\{d_{1}, d_{2}, \cdots, d_{N}\right\} \in R^{N \times N}$. In the diagonal matrix, $d_{i}$ is the in-degree of $v_{i}$, formulated by $d_{i}=\sum_{j=1}^{N} a_{i j}(i=1,2, \cdots, N)$. Accordingly, the Laplacian matrix of $G$ can be defined by $L=D-A \in R^{N \times N}$. Proven in [13], $L$ has at least one zero eigenvalue; all other eigenvalues are located at the open right-half plane if $G$ is connected.

Assumption 3: $G$ of the multi-agent system has a spanning tree, indicating that the zero eigenvalue of $L$ is simple.

Assumption 4: In the multi-agent system, the sole leader cannot receive any information from the followers.

Consider Assumption 3. For the zero eigenvalue, an eigenvector of $L$ is $\mathbf{1}_{N}$, that is, $L \mathbf{1}_{N}=\mathbf{0}_{N}$ holds true, where $\mathbf{1}_{N}=[1,1, \cdots, 1]^{T} \in R^{N \times 1}$ and $\mathbf{0}_{N}=[0,0, \cdots, 0]^{T} \in R^{N \times 1}$. Further, $\operatorname{rank}(L)=N-1$ for the simple zero eigenvalue [13].

Without loss of generality, the $N$ th agent in the multi-agent system is named leader and other $N-1$ agents are followers. Consider Assumption 4, that is, $a_{N i}=0(i=1,2, \cdots, N)$ and the Laplacian matrix of $G$ can be written as

$$
L=\left[\begin{array}{ccccc}
d_{1} & -a_{12} & \cdots & -a_{1(N-1)} & -a_{1 N} \\
-a_{21} & d_{2} & \cdots & -a_{2(n-1)} & -a_{2 N} \\
\vdots & \vdots & \ddots & \vdots & \vdots \\
-a_{(N-1) 1} & -a_{(N-2) 2} & \cdots & d_{N-1} & -a_{(N-1) N} \\
0 & 0 & 0 & 0 & 0
\end{array}\right]
$$


Further, the communication topologies among all the followers are described by a directed graph $\bar{G}$. Apparently, $\bar{G}$ is a subgraph of $G$. The weighted adjacency matrix $\bar{A} \in R^{(N-1) \times(N-1)}$ of $\bar{G}$ is defined by

$$
\bar{A}=\left[\begin{array}{cccc}
a_{11} & a_{12} & \cdots & a_{1(N-1)} \\
a_{21} & a_{22} & \cdots & a_{2(N-1)} \\
\vdots & \vdots & \ddots & \vdots \\
a_{(N-1) 1} & a_{(N-1) 2} & \cdots & a_{(N-1)(N-1)}
\end{array}\right]
$$

The degree matrix of $\bar{G}$ is determined by $\overline{\mathscr{D}}=\operatorname{diag}\left\{\bar{d}_{1}, \overline{d_{2}}\right.$, $\left.\cdots, \bar{d}_{N-1}\right\}$, where $\bar{d}_{i}=\sum_{j=1}^{N-1} a_{i j}(i=1,2, \cdots, N-1)$. Accordingly, the Laplacian matrix of $\bar{G}$ can be defined as $\bar{D}-\bar{A}$, formulated by

$$
\bar{L}=\left[\begin{array}{cccc}
\overline{d_{1}} & -a_{12} & \cdots & -a_{1(N-1)} \\
-a_{21} & \overline{d_{2}} & \cdots & -a_{2(N-2)} \\
\vdots & \vdots & \ddots & \vdots \\
-a_{(N-1) 1} & -a_{(N-1) 2} & \cdots & \bar{d}_{N-1}
\end{array}\right]
$$

Similarly, assuming that the subgraph $\bar{G}$ is itself a directed graph, $\bar{L} \mathbf{1}_{N-1}=\mathbf{0}_{N-1}$ exists where $\mathbf{1}_{N-1}=[1,1, \cdots, 1]^{T} \in$ $\in R^{(N-1) \times 1}$ and $\mathbf{0}_{N-1}=[0,0, \cdots, 0]^{T} \in R^{(N-1) \times 1}$. Moreover, define a matrix ${ }^{-}=\operatorname{diag}\left\{b_{1}, b_{2}, \cdots, b_{N-1}\right\}$, where $\bar{B}=\operatorname{diag}\left\{\bar{b}_{1}, \bar{b}_{2}, \cdots\right.$, $\left.\bar{b}_{N-1}\right\}$. Apparently, there exists $\operatorname{rank}(\bar{L}+\bar{B})=\operatorname{rank}(L)=$ $=N-1$.

\section{Formation design}

Assumption 5: Considering the formation-control problem of the multi-agent system, the leader is assumed to be well controlled by a developed technology; the controller's and the leader's dynamic is known [17].

The $N$ th agent identified as the leader has to track a predefined trajectory and its control problem can be treated as the tracking-control problem of a single robot. Concerning Assumption 5, the leader can be treated as a nominal one in the formation-control problem, that is, $\delta_{x N}=\delta_{y N}=0$. The other $N-1$ agents act as followers and they are equipped with the designed formation controllers to achieve formation maneuvers of the multi-agent system.

Concerning the $i$ th follower agent $(i=1,2, \cdots, N-1)$, its equations of motion in (8) are decoupled in the $x$-axis and $y$-axis. Consequently, its formation design can be divided into two parts, that is, design for the $x$-axis and design for the $y$-axis. Here the formation design for the $x$-axis is taken into account first. From (8), the $x$-axis subsystem with uncertainties can be written as

$$
\left[\begin{array}{c}
\dot{x}_{h i} \\
\dot{v}_{x i}
\end{array}\right]=\left[\begin{array}{c}
v_{x i} \\
u_{x i}+\delta_{x i}
\end{array}\right]
$$

Further, (13) can be re-written by the following state-space representation.

$$
\dot{\mathbf{x}}_{x i}=\mathbb{A}_{x i} \mathbf{x}_{x i}+\mathbb{B}_{x i} u_{x i}+\mathbb{B}_{x i} \delta_{x i}
$$

where $\mathbf{x}_{x i}=\left[\begin{array}{ll}x_{h i} & v_{x i}\end{array}\right]^{T}, \mathbb{A}_{x i}=\left[\begin{array}{ll}0 & 1 \\ 0 & 0\end{array}\right], \mathbb{B}_{x i}=\left[\begin{array}{ll}0 & 0\end{array}\right]^{T}$ and $u_{x i}$ is the
control input.

3.1. NDO-based observer design. Consider the $x$-axis subsystem (14) and design its NDO-based observer (15) [33, 34].

$\left\{\begin{array}{l}\dot{p}_{x i}=-\mathbb{L}_{x i}^{T} \mathbb{B}_{x i} p_{x i}-\mathbb{L}_{x i}^{T}\left(\mathbb{B}_{x i} \mathbb{L}_{x i}^{T} \mathbf{x}_{x i}+\mathbb{A}_{x i} \mathbf{x}_{x i}+\mathbb{B}_{x i} u_{x i}\right) \\ \hat{\delta}_{x i}=p_{x i}+\mathbb{L}_{x i}^{T} \mathbf{x}_{x i}\end{array}\right.$

In (15), $p_{x i}$ is the internal state variable of the observer, $\hat{\delta}_{x i}$ is the approximation of $\delta_{x i}$ and the gain vector $\mathbb{L}_{x i} \in R^{2 \times 1}$ is designed such that the constant $\lambda_{x i}=\mathbb{L}_{x i}^{T} \mathbb{B}_{x i}$ is positive.

Define an estimation-error variable $e_{x i_{d}}=\delta_{x i}-\hat{\delta}_{x i}$, differentiate the error variable with respect to time $t$, substitute (15) into the derivative of $e_{x i_{d}}$ and take Assumptions 1 and 2 into account. We can obtain (16).

$$
\begin{aligned}
\dot{e}_{x i}= & \dot{\delta}_{x i}-\dot{\hat{\delta}}_{x i} \simeq-\dot{p}_{x i}-\mathbb{L}_{x i}^{T} \dot{\mathbf{x}}_{x i} \\
= & \lambda_{x i} p_{x i}+\mathbb{L}_{x i}^{T}\left(\mathbb{B}_{x i} \mathbb{L}_{x i}^{T} \mathbf{x}_{x i}+\mathbb{A}_{x i} \mathbf{x}_{x i}+\mathbb{B}_{x i} u_{x i}\right) \\
& -\mathbb{L}_{x i}^{T}\left(\mathbb{A}_{x i} \mathbf{x}_{x i}+\mathbb{B}_{x i} u_{x i}+\mathbb{B}_{x i} \delta_{x i}\right) \\
= & \lambda_{x i}\left(\hat{\delta}_{x i}-\mathbb{L}_{x i}^{T} \mathbf{x}_{x i}\right)+\mathbb{L}_{x i}^{T}\left(\mathbb{B}_{x i} \mathbb{L}_{x i}^{T} \mathbf{x}_{x i}+\mathbb{A} \mathbf{x}_{x i}+\mathbb{B} u_{x i}\right) \\
& -\mathbb{L}_{x i}^{T}\left(\mathbb{A}_{x i} \mathbf{x}_{x i}+\mathbb{B}_{x i} u_{x i}+\mathbb{B}_{x i} \delta_{i k}\right) \\
= & \lambda_{x i}\left(\hat{\delta}_{x i}-\delta_{x i}\right)=-\lambda_{x i} e_{x i}
\end{aligned}
$$

The solution of (16) is $e_{x i_{d}}=\exp \left(-\lambda_{x i} t\right) e_{x i_{d}}(0)$, where $e_{x i_{d}}(0)$ is the initial condition at $t=0$. Owing to $\lambda_{x i}>0$, this fact indicates that the estimation-error variable $e_{x i_{d}}$ is exponentially convergent to 0 as $t \rightarrow \infty$.

3.2. Integral SMC-based controller design. To coordinate the leader-following-based multi-agent system, a tracking-error variable of the $i$ th follower agent is defined by

$$
\begin{aligned}
e_{x i}= & \sum_{j=1}^{N-1} a_{i j}\left[\left(x_{h i}-x_{h j}-d_{i j}^{x}\right)+\rho_{x i}\left(v_{x i}-v_{x j}\right)\right] \\
& +\bar{b}_{i}\left(x_{h i}-x_{h N}-d_{i N}^{x}\right)+\bar{b}_{i} \rho_{x i}\left(v_{x i}-v_{x N}\right)
\end{aligned}
$$

where $\rho_{x i}>0$ is a pre-defined constant, $d_{i j}^{x}$ is the pre-defined relative position between the $i$ th follower and the $j$ th follower and $d_{i N}^{x}$ is the pre-defined relative position between the $i$ th follower and the leader.

Differentiate $e_{x i}$ in (17) with respect to time $t$, substitute the $x$-axis subsystem (13) into the derivative of $e_{x i}$ and consider Assumption 5 to obtain 


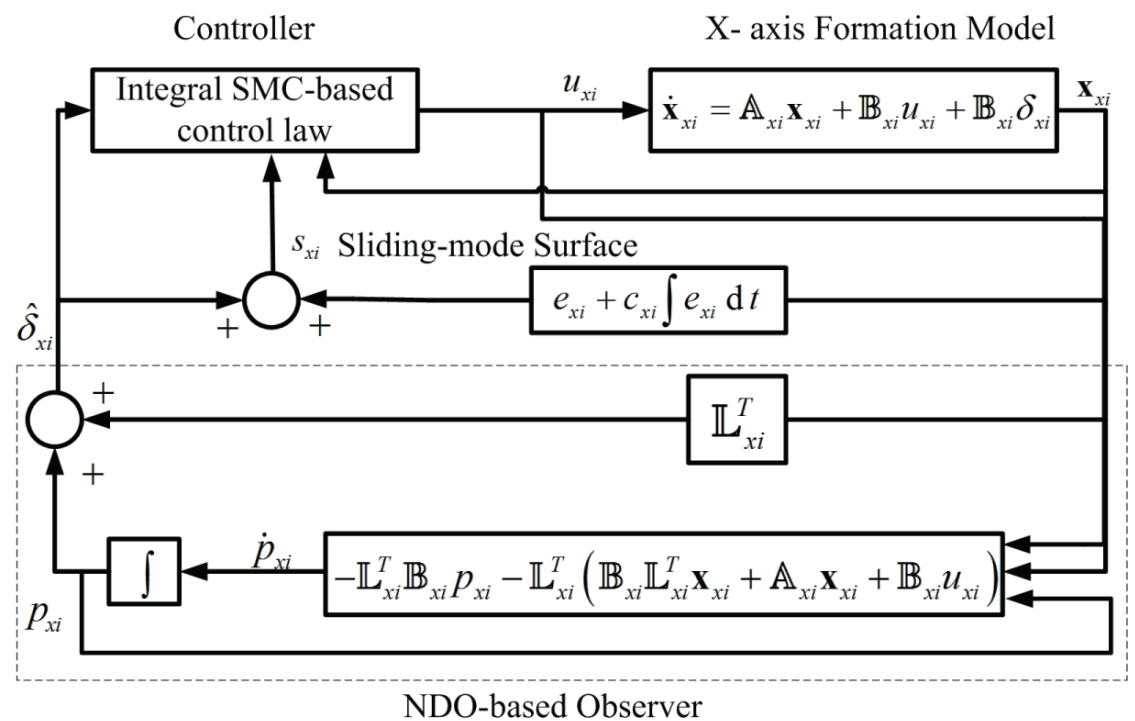

Fig. 2. Structure of the control scheme

$$
\begin{aligned}
\dot{e}_{x i}= & \sum_{j=1}^{N-1} a_{i j}\left[\left(v_{x i}-v_{x j}\right)+\rho_{x i}\left(u_{x i}-u_{x j}\right)+\rho_{x i}\left(\delta_{x i}-\delta_{x j}\right)\right] \\
& +\bar{b}_{i}\left[\left(v_{x i}-v_{x N}\right)+\rho_{x i}\left(u_{x i}-u_{x N}\right)+\rho_{x i} \delta_{x i}\right]
\end{aligned}
$$

Concerning the $x$-axis subsystem of the $i$ th agent, an integral sliding-mode surface with the NDO-based observer output [11] is defined by

$$
s_{x i}=e_{x i}+c_{x i} \int e_{x i} \mathrm{~d} t+\hat{\delta}_{x i}
$$

where $c_{x i}>0$ is constant.

The derivative of the sliding-mode surface variable with respect to time $t$ can be written by

$$
\dot{s}_{x i}=\dot{e}_{x i}+c_{x i} e_{x i}+\dot{\hat{\delta}}_{x i}
$$

Substituting (18) and (19) into (20) gives

$$
\begin{aligned}
\dot{s}_{x i}= & \sum_{j=1}^{N-1} a_{i j}\left[\left(v_{x i}-v_{x j}\right)+\rho_{x i}\left(u_{x i}-u_{x j}\right)+\rho_{x i}\left(\delta_{x i}-\delta_{x j}\right)\right] \\
& +\bar{b}_{i}\left[\left(v_{x i}-v_{x N}\right)+\rho_{x i}\left(u_{x i}-u_{x N}\right)+\rho_{x i} \delta_{x i}\right] \\
& +c_{x i} \sum_{j=1}^{N-1} a_{i j}\left[\left(x_{h i}-x_{h j}-d_{i j}^{x}\right)+\rho_{x i}\left(v_{x i}-v_{x j}\right)\right] \\
& +\bar{b}_{i} c_{x i}\left(x_{h i}-x_{h N}-d_{i N}^{x}\right)+\bar{b}_{i} c_{x i} \rho_{x i}\left(v_{x i}-v_{x N}\right)+\dot{\hat{\delta}}_{x i}
\end{aligned}
$$

Design the following $x$-axis formation-control law for the $i$ th follower agent

$$
\begin{aligned}
u_{x i}= & -\frac{c_{x i} \rho_{x i}+1}{\rho_{x i}\left(d_{i}+b_{i}\right)} \sum_{j=1}^{N-1} a_{i j}\left(v_{x i}-v_{x j}\right) \\
& -\frac{c_{x i} \rho_{x i}+1}{\rho_{x i}\left(d_{i}+b_{i}\right)} \bar{b}_{i}\left(v_{x i}-v_{x N}\right) \\
& -\frac{\bar{b}_{i}}{d_{i}+b_{i}} \hat{\delta}_{x i}-\frac{1}{d_{i}+b_{i}} \sum_{j=1}^{N-1} a_{i j}\left(\hat{\delta}_{x i}-\hat{\delta}_{x j}\right) \\
& -\frac{c_{x i}}{\rho_{x i}\left(d_{i}+b_{i}\right)} \sum_{j=1}^{N-1} a_{i j}\left(x_{h i}-x_{h j}-d_{i j}^{x}\right) \\
& -\frac{\bar{b}_{i} c_{x i}}{\rho_{x i}\left(d_{i}+b_{i}\right)}\left(x_{h i}-x_{h N}-d_{i N}^{x}\right)+\frac{1}{d_{i}+b_{i}} \sum_{j=1}^{N-1} a_{i j} u_{x j} \\
& -\frac{\kappa_{x i}}{\rho_{x i}\left(d_{i}+b_{i}\right)} \operatorname{sgn}\left(s_{x i}\right)
\end{aligned}
$$

where $\kappa_{x i}>0$ is predefined and $\operatorname{sgn}(\cdot)$ is the sign function.

The equation $\dot{\hat{\delta}}_{x i}=-\lambda_{x i}\left(\hat{\delta}_{x i}-\delta_{x i}\right)$ can be drawn from (16). Replace $\hat{\delta}_{x i}$ in (21) with the equation and replace $u_{x i}$ in (21) with (22). Then, $\dot{s}_{x i}$ can be re-arranged

$$
\begin{aligned}
\dot{s}_{x i}= & \rho_{x i}\left(\overline{d_{i}}+\bar{b}_{i}\right) e_{x i_{d}}-\rho_{x i} \sum_{j=1}^{N-1} a_{i j} e_{x j_{d}} \\
& -\kappa_{x i} \operatorname{sgn}\left(s_{x i}\right)-\lambda_{x i}\left(\hat{\delta}_{x i}-\delta_{x i}\right)
\end{aligned}
$$

3.3. System structure. The system structure is presented in Fig. 2. Concerning the $i$ th follower agent, its state-variable vector of the $x$-axis subsystem $\mathbf{x}_{x i}$, composed of $x_{h i}$ and $v_{x i}$, is 
located at the feedback channel. As shown in Fig. 2, it feeds on the NDO-based observer to estimate the approximation $\hat{\delta}_{x i}$. Furthermore, the tracking-error variable $e_{x i}$ and its integral construct the integral sliding-mode surface variable $s_{x i}$. The designed control scheme employs $s_{x i}, \mathbf{x}_{x i}$ and $\hat{\delta}_{x i}$ to generate the final control input $u_{x i}$. Finally, $u_{x i}$ is applied to the $i$ th follower agent to achieve formation maneuvers of its $x$-axis subsystem.

3.4. Stability analysis. In order to consider the formation stability of the $x$-axis subsystem, the following assumption about the estimation-error variable is considered.

Assumption 6: $\left|e_{x i_{d}}\right|<e_{x i_{d}}$, where $e_{x i_{d}}^{*}>0$ is constant but unknown.

Theorem 1: Concerning the $i$ th follower agent, consider its $x$-axis subsystem dynamic (13), take Assumptions 1-6 into account, design the NDO-based observer (15), define the sliding-mode surface (19) and utilize the integral SMC-based control law (22). The closed-loop control system of the $x$-axis subsystem structured in Fig. 2 is asymptotically stable if $\kappa_{x i}>\left(\lambda_{x i}+\rho_{x i} \bar{b}_{i}\right) e_{x i_{d}}^{*}$.

Proof: Select a Lyapunov candidate function $V=\frac{1}{2} s_{x i}^{2}$. Differentiate $V$ with respect to time $t$. The derivative of $V$ can be written as $\dot{V}=s_{x i} \dot{s}_{x i}$. Replace $\dot{S}_{x i}$ by (23). The derivative of $V$ has the form of

$$
\begin{aligned}
\dot{V} & =s_{x i}\left[\rho_{x i}\left(\overline{d_{i}}+\overline{b_{i}}\right) e_{x i_{d}}-\rho_{x i} \sum_{j=1}^{N-1} a_{i j} e_{x j_{d}}-\right. \\
& \left.-\kappa_{x i} \operatorname{sgn}\left(s_{x i}\right)-\lambda_{x i}\left(\hat{\delta}_{x i}-\delta_{x i}\right)\right] \\
& =s_{x i}\left[\rho_{x i}\left(\overline{d_{i}}+\bar{b}_{i}\right) e_{x i_{d}}-\rho_{x i} \sum_{j=1}^{N-1} a_{i j} e_{x i_{d}}-\right. \\
& \left.-\kappa_{x i} \operatorname{sgn}\left(s_{x i}\right)+\lambda_{x i} e_{x i_{d}}\right] \\
& =-\kappa_{x i}\left|s_{x i}\right|+\left[\lambda_{x i} e_{x i_{d}}+\rho_{x i}\left(\overline{d_{i}}+\bar{b}_{i}\right) e_{x i_{d}}-\right. \\
& \left.-\rho_{x i} \sum_{j=1}^{N-1} a_{i j} e_{x i}\right] s_{x i} \\
& \leq-\kappa_{x i}\left|s_{x i}\right|+\left[\lambda_{x i} e_{x i_{d}}^{*}+\rho_{x i}\left(\overline{d_{i}}+\bar{b}_{i}\right) e_{x i_{d}}^{*}-\right. \\
& \left.-\rho_{x i} \overline{d_{i}} e_{x i_{d}}^{*}\right] s_{x i} \\
& =-\kappa_{x i}\left|s_{x i}\right|+\left(\lambda_{x i}+\rho_{x i} \overline{b_{i}}\right) e_{x i_{d}}^{*} s_{x i} \\
& \leq\left[-\kappa_{x i}+\left(\lambda_{x i}+\rho_{x i} \bar{b}_{i}\right) e_{x i_{d}}^{*}\right]\left|s_{x i}\right|
\end{aligned}
$$

Pick up $\kappa_{x i}>\left(\lambda_{x i}+\rho_{x i} \bar{b}_{i}\right) e_{x i_{d}}^{*}$ such that $\dot{V}<0$ exists. Concerning $V \geq 0$, the closed-loop control system of the $x$-axis subsystem is asymptotically stable in Lyapunov sense.
Furthermore, the closed-loop stability of the $x$-axis subsystem can be extended to the multi-agent system. Concerning the $i$ th follower agent, define $\mathbf{z}_{i}=\left[\begin{array}{ll}x_{h i} & y_{h i}\end{array}\right]^{T} \in R^{2 \times 1}$ such that the augmented vector of $\mathbf{z}_{i}$ in the multi-agent system can be written as $\mathbf{z}=\left[\begin{array}{lll}\mathbf{z}_{1}^{T} & \mathbf{z}_{2}^{T} \cdots & \mathbf{z}_{N-1}^{T}\end{array}\right]^{T} \in R^{2(N-1) \times 1}$.

Similarly, define the following vectors $\mathbf{v}_{i}=\left[\begin{array}{ll}v_{x i} & v_{y i}\end{array}\right]^{T}, \mathbf{u}_{i}=$ $=\left[u_{x i} u_{y i}\right]^{T}, \Delta_{i}=\left[\delta_{x i} \delta_{y i}\right]^{T}, \hat{\Delta}_{i}=\left[\hat{\delta}_{x i} \hat{\delta}_{y i}\right]^{T}, \mathbf{e}_{i}=\left[e_{x i} e_{y i}\right]^{T}, \mathbf{e}_{i_{d}}=\left[e_{x i_{d}} e_{y i_{d}}\right]^{T}$, $\mathbf{d}_{i j}=\left[\begin{array}{ll}d_{i j}^{x} & d_{i j}^{y}\end{array}\right]^{T}, \mathbf{d}_{i N}=\left[d_{i N}^{x} d_{i N}^{y}\right]^{T}$ and $\mathbf{s}_{i}=\left[s_{x i} s_{y i}\right]^{T}$. Here $\mathbf{v}_{i}, \mathbf{u}_{i}, \Delta_{i}, \hat{\Delta}_{i}, \mathbf{e}_{i}$, $\mathbf{e}_{i_{d}}, \mathbf{d}_{i j}, \mathbf{d}_{i N}$ and $\mathbf{s}_{i} \in R^{2 \times 1}$. Correspondingly, their augmented vectors

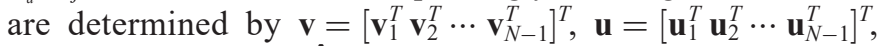
$\Delta=\left[\Delta_{1}^{T} \Delta_{2}^{T} \cdots \Delta_{N-1}^{T}\right]^{T}, \hat{\Delta}=\left[\hat{\Delta}_{1}^{T} \hat{\Delta}_{2}^{T} \cdots \hat{\Delta}_{N-1}^{T}\right]^{T}, \mathbf{e}=\left[\mathbf{e}_{1}^{T} \mathbf{e}_{2}^{T} \cdots \mathbf{e}_{N-1}^{T}\right]^{T}$,

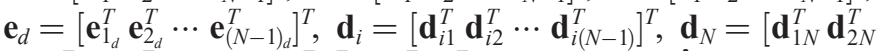
$\left.\cdots \mathbf{d}_{(N-1) N}^{T}\right]^{T}$ and $\mathbf{s}=\left[\mathbf{s}_{1}^{T} \mathbf{s}_{2}^{T} \cdots \mathbf{s}_{N-1}^{T}\right]^{T}$. Here $\mathbf{v}, \mathbf{u}, \Delta, \hat{\Delta}, \mathbf{e}, \mathbf{e}_{d}, \mathbf{d}_{i}, \mathbf{d}_{N}$ and $\mathbf{s} \in R^{2(N-1) \times 1}$.

Define $\Upsilon=\operatorname{diag}\left\{\rho_{x 1}, \rho_{y 1}, \cdots, \rho_{x(N-1)}, \rho_{y(N-1)}\right\}, \mathbf{c}=\operatorname{diag}\left\{c_{x 1}\right.$, $\left.c_{y 1}, \cdots, c_{x(N-1)}, c_{y(N-1)}\right\}, \Lambda=\operatorname{diag}\left\{\lambda_{x 1}, \lambda_{y 1}, \cdots, \lambda_{x(n-1)}, \lambda_{y(n-1)}\right\}$ and $\kappa=\operatorname{diag}\left\{\kappa_{x 1}, \kappa_{y 1}, \cdots, \kappa_{x(N-1)}, \kappa_{y(N-1)}\right\}$. Here $\Upsilon, \mathbf{c}, \Lambda$ and $\kappa \in R^{2(N-1) \times 2(N-1)}$.

From the above vectors and matrices, the augmented tracking-error vector e can be written as

$$
\begin{aligned}
\mathbf{e} & =\left[(\bar{L}+\bar{B}) \otimes \mathbf{I}_{2}\right]\left(\mathbf{z}-\mathbf{d}_{i}\right)+\Upsilon\left[(\bar{L}+\bar{B}) \otimes \mathbf{I}_{2}\right] \mathbf{v} \\
& -\left(\bar{B} \otimes \mathbf{I}_{2}\right]\left(\mathbf{1}_{N-1} \otimes \mathbf{z}_{N}-\mathbf{d}_{N}\right)-\Upsilon\left(\bar{B} \mathbf{1}_{N-1} \otimes \mathbf{I}_{2}\right) \mathbf{v}_{n}
\end{aligned}
$$

where $\mathbf{I}_{2}$ is a $2 \times 2$ identity matrix and $\oplus$ means the Kronecker product. Both of $\mathbf{z}_{N}$ and $\mathbf{v}_{N}$ are concerned to the leader agent, determined by $\mathbf{z}_{N}=\left[\begin{array}{ll}x_{h N} & y_{h N}\end{array}\right]^{T}$ and $\mathbf{v}_{N}=\left[\begin{array}{ll}v_{x N} & v_{y N}\end{array}\right]^{T}$.

Differentiating $\mathbf{e}$ in (25) with respect to time $t$ gives

$$
\begin{aligned}
\dot{\mathbf{e}} & =\left[(\bar{L}+\bar{B}) \otimes \mathbf{I}_{2}\right] \mathbf{v}+\Upsilon\left[(\bar{L}+\bar{B}) \otimes \mathbf{I}_{2}\right] \mathbf{u} \\
& +\Upsilon\left[(\bar{L}+\bar{B}) \otimes \mathbf{I}_{2}\right] \Delta-\left(\bar{B} \mathbf{1}_{N-1} \otimes \mathbf{I}_{2}\right) \mathbf{v}_{N} \\
& -\Upsilon\left(\bar{B} \mathbf{1}_{N-1} \otimes \mathbf{I}_{2}\right) \mathbf{u}_{N}
\end{aligned}
$$

where $\mathbf{u}_{N}$ is formulated by $\mathbf{u}_{N}=\left[\begin{array}{ll}u_{x N} & u_{y N}\end{array}\right]^{T}$.

The augmented integral sliding-mode surface vector is formulated by

$$
\mathbf{s}=\mathbf{e}+\mathbf{c} \int \mathbf{e d} t+\hat{\Delta}
$$

Differentiating $\mathbf{s}$ in (27) with respect to time $t$ yields

$$
\begin{aligned}
\dot{\mathbf{s}} & =\dot{\mathbf{e}}+\mathbf{c e}+\dot{\hat{\Delta}} \\
& =\left[(\bar{L}+\bar{B}) \otimes \mathbf{I}_{2}\right] \mathbf{v}+\Upsilon\left[(\bar{L}+\bar{B}) \otimes \mathbf{I}_{2}\right] \mathbf{u} \\
& +\Upsilon\left[(\bar{L}+\bar{B}) \otimes \mathbf{I}_{2}\right] \Delta-\left(\bar{B} \mathbf{1}_{N-1} \otimes \mathbf{I}_{2}\right) \mathbf{v}_{N} \\
& -\Upsilon\left(\bar{B} \mathbf{1}_{N-1} \otimes \mathbf{I}_{2}\right) \mathbf{u}_{N}+\mathbf{c}\left[(\bar{L}+\bar{B}) \otimes \mathbf{I}_{2}\right]\left(\mathbf{z}-\mathbf{d}_{i}\right) \\
& -\mathbf{c} \Upsilon\left[(\bar{L}+\bar{B}) \otimes \mathbf{I}_{2}\right] \mathbf{v}+\mathbf{c} \Upsilon\left(\bar{B} \mathbf{1}_{N-1} \otimes \mathbf{I}_{2}\right) \mathbf{v}_{N} \\
& -\mathbf{c}\left(\bar{B} \otimes \mathbf{I}_{2}\right)\left(\mathbf{1}_{N-1} \otimes \mathbf{z}_{N}-\mathbf{d}_{N}\right)+\dot{\hat{\Delta}}
\end{aligned}
$$


Design the control law $\mathbf{u}$ of the multi-agent system as

$$
\begin{aligned}
\mathbf{u} & =\Upsilon^{-1}\left[(\bar{L}+\bar{B}) \otimes \mathbf{I}_{2}\right]^{-1}\left\{\mathbf{c}\left[(\bar{L}+\bar{B}) \otimes \mathbf{I}_{2}\right](\mathbf{z}-\mathbf{d})+\right. \\
& +\left(\mathbf{I}_{2(N-1)}+\mathbf{c} \Upsilon\right)\left[(\bar{L}+\bar{B}) \otimes \mathbf{I}_{2}\right] \mathbf{v}- \\
& -\left(\mathbf{I}_{2(N-1)}+\mathbf{c} \Upsilon\right)\left(\bar{B} \mathbf{1}_{N-1} \otimes \mathbf{I}_{2}\right) \mathbf{v}_{N}- \\
& -\mathbf{c}\left(\bar{B} \otimes \mathbf{I}_{2}\right)\left(\mathbf{1}_{N-1} \otimes \mathbf{z}_{N}-\mathbf{d}_{N}\right)-\Upsilon\left(\bar{B} \mathbf{1}_{N-1} \otimes \mathbf{I}_{2}\right) \mathbf{u}_{N}+ \\
& \left.+\Upsilon\left[(\bar{L}+\bar{B}) \otimes \mathbf{I}_{2}\right] \hat{\Delta}+\kappa \operatorname{sgn}(\mathbf{s})\right\}
\end{aligned}
$$

In (29), $\mathbf{I}_{2(N-1)}$ is a $2(N-1) \times 2(N-1)$ identity matrix. Replacing the control law $\mathbf{u}$ in (28) by (29) gives

$$
\dot{\mathbf{s}}=\Upsilon\left[(\bar{L}+\bar{B}) \otimes \mathbf{I}_{2}\right](\Delta-\hat{\Delta})-\kappa \operatorname{sgn}(\mathbf{s})+\dot{\hat{\Delta}}
$$

Theorem 2: Concern the multi-agent system, suppose that its communication graph has a directed spanning tree. The stability of the leader-following formation control is guaranteed if the controller parameters of each follower agent are designed by Theorem 1.

Proof: Define a Lyapunov candidate function $V^{\prime}(t)=\|\mathbf{s}\|_{2}$ here $\|\cdot\|_{2}$ means 2-norm. Differentiate $V^{\prime}(t)$ with respect to time $t$. The derivative of $V^{\prime}$ can be written by $\dot{V}^{\prime}(t)=\frac{\mathbf{s}^{T} \dot{\mathbf{s}}}{\|\mathbf{s}\|_{2}}$. Replacing $\dot{\mathbf{s}}$ in the derivative of $V^{\prime}$ by (30) yields

$$
\begin{aligned}
\dot{V}^{\prime}= & \frac{\mathbf{s}^{T}}{\|\mathbf{s}\|_{2}}\left\{\Upsilon\left[(\bar{L}+\bar{B}) \otimes \mathbf{I}_{2}\right](\Delta-\hat{\Delta})-\kappa \operatorname{sgn}(\mathbf{s})+\dot{\hat{\Delta}}\right\} \\
\simeq & \frac{\mathbf{s}^{T}}{\|\mathbf{s}\|_{2}}\left\{\Upsilon\left[(\bar{L}+\bar{B}) \otimes \mathbf{I}_{2}\right] \mathbf{e}_{d}-\kappa \operatorname{sgn}(\mathbf{s})+\Lambda \mathbf{e}_{d}\right\} \\
\leq & -\|\kappa\|_{1} \frac{\mathbf{s}^{T} \operatorname{sgn}(\mathbf{s})}{\|\mathbf{s}\|_{2}}+\|\Lambda\|_{1} \frac{\mathbf{s}^{T} \mathbf{e}_{d}}{\|\mathbf{s}\|_{2}} \\
& +\|\Upsilon\|_{1} \frac{\mathbf{s}^{T}\left[(\bar{L}+\bar{B}) \otimes \mathbf{I}_{2}\right] \mathbf{e}_{d}}{\|\mathbf{s}\|_{2}} \\
\leq & -\|\kappa\|_{1} \frac{\|\mathbf{s}\|_{1}}{\|\mathbf{s}\|_{2}}+\|\Lambda\|_{1} \frac{\mathbf{s}^{T} \mathbf{1}_{2(N-1)}}{\|\mathbf{s}\|_{2}} e_{d}^{*} \\
& +\|\Upsilon\|_{1} \frac{\mathbf{s}^{T}\left[(\bar{L}+\bar{B}) \otimes \mathbf{I}_{2}\right] \mathbf{1}_{2(N-1)}}{\|\mathbf{s}\|_{2}} e_{d}^{*}
\end{aligned}
$$

In (31), $e_{d}^{*}=\left\|\mathbf{e}_{d}\right\|_{\infty}$ where $\|\cdot\|_{\infty}$ means $\infty$-norm. Note that $a_{i i}=0(i=1, \cdots, N-1)$ in (11) such that $\left[(\bar{L}+\bar{B}) \oplus \mathbf{I}_{2}\right]$ $\mathbf{1}_{2(N-1)}=\left[\bar{b}_{1} \bar{b}_{1} \cdots \bar{b}_{N-1} \bar{b}_{N-1}\right]^{T} \in R^{2(N-1)}$. Let $b^{*}=\max \left\{\bar{b}_{1}, \cdots\right.$, $\left.\bar{b}_{N-1}\right\}=\|B\|_{\infty}$. (31) can be re-arranged

$$
\begin{aligned}
\dot{V}^{\prime} \leq & -\|\kappa\|_{1} \frac{\|\mathbf{s}\|_{1}}{\|\mathbf{s}\|_{2}}+\|\Lambda\|_{1} \frac{\mathbf{s}^{T} \mathbf{1}_{2(N-1)}}{\|\mathbf{s}\|_{2}} e_{d}^{*} \\
& +\|\Upsilon\|_{1} \frac{\mathbf{s}^{T} \mathbf{1}_{2(N-1)}}{\|\mathbf{s}\|_{2}} b^{*} e_{d}^{*} \\
\leq & -\|\kappa\|_{1} \frac{\|\mathbf{s}\|_{1}}{\|\mathbf{s}\|_{2}}+\|\Lambda\|_{1} \frac{\|\mathbf{s}\|_{1}}{\|\mathbf{s}\|_{2}} e_{d}^{*}+\|\Upsilon\|_{1} \frac{\|\mathbf{s}\|_{1}}{\|\mathbf{s}\|_{2}} b^{*} e_{d}^{*}
\end{aligned}
$$

If the controller parameters of each follower agent are selected by Theorem $1, \dot{V}^{\prime}<0$ can be deduced from (32). Con- sidering $V^{\prime} \geq 0$, the formation control of the multi-agent system is asymptotically stable in Lyapunov sense.

Take Assumption 6 into account. The tracking-error variable $e_{x i_{d}}^{*}$ is constant but unknown, meaning that it is hard to determine $\kappa_{i k}$ in Theorem 1 as well as $\kappa$ in Theorem 2. To guarantee the formation stability, a conservative value of $e_{x i_{d}}^{*}$ should be assigned. In this aspect, there seem no benefits earned from such an NDO-based integral SMC scheme. However, $e_{x i_{d}}^{*}$ originated from the scheme is exponentially convergent as proven, indicating that a small value of $e_{x i_{d}}^{*}$ could be chosen. According to Theorem 1, this kind of formation design could contribute to the decrease of the chattering phenomenon, as well as the improvement of the formation performance.

\section{Simulation results}

To demonstrate the performance of the presented control scheme, this section implements some simulations on a multi-robot platform and discusses the results. The platform is composed of four mobile wheeled robots. The robots are identical (Fig. 1), with three follower agents in order marked as 1,2 and 3 and the sole leader agent numbered as 4 . The physical parameters of these agents are picked up from [16], listed by $l=0.0265 \mathrm{~m}$, $h=0.04 \mathrm{~m}, \quad r=0.02 \mathrm{~m}, m_{b}=0.018 \mathrm{~kg}, m_{w}=0.007 \mathrm{~kg}$, $I_{b}=1.44 \times 10^{-4} \mathrm{~kg} \cdot \mathrm{m}^{2}$ and $I_{w}=1.44 \times 10^{-6} \mathrm{~kg} \cdot \mathrm{m}^{2}$. The communication topology of this multi-agent system is illustrated in Fig. 3.

As shown in Fig. 3, the communication graph $G$ forms a standard spanning tree, where the adjacency and Laplacian matrices are formulated by

$$
A=\left[\begin{array}{llll}
0 & 1 & 0 & 1 \\
1 & 0 & 0 & 0 \\
0 & 0 & 0 & 1 \\
0 & 0 & 0 & 0
\end{array}\right] \text { and } L=\left[\begin{array}{cccc}
2 & -1 & 0 & -1 \\
-1 & 1 & 0 & 0 \\
0 & 0 & 1 & -1 \\
0 & 0 & 0 & 0
\end{array}\right]
$$

Further, the communication subgraph $\overline{\mathscr{G}}$ can be derived from $\mathscr{G}$, whose adjacency and Laplacian matrices are determined by

$$
\bar{A}=\left[\begin{array}{lll}
0 & 1 & 0 \\
1 & 0 & 0 \\
0 & 0 & 0
\end{array}\right] \text { and } \bar{L}=\left[\begin{array}{ccc}
2 & -1 & 0 \\
-1 & 1 & 0 \\
0 & 0 & 1
\end{array}\right]
$$

Apparently, the subgraph $\bar{G}$ is itself a directed graph.

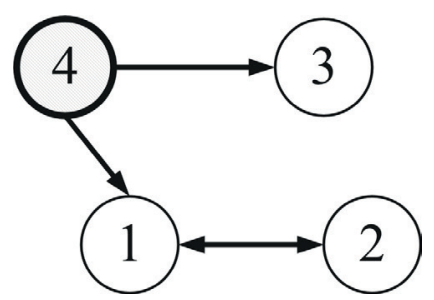

Fig. 3. Communication topology of the multi-agent platform 
(a)

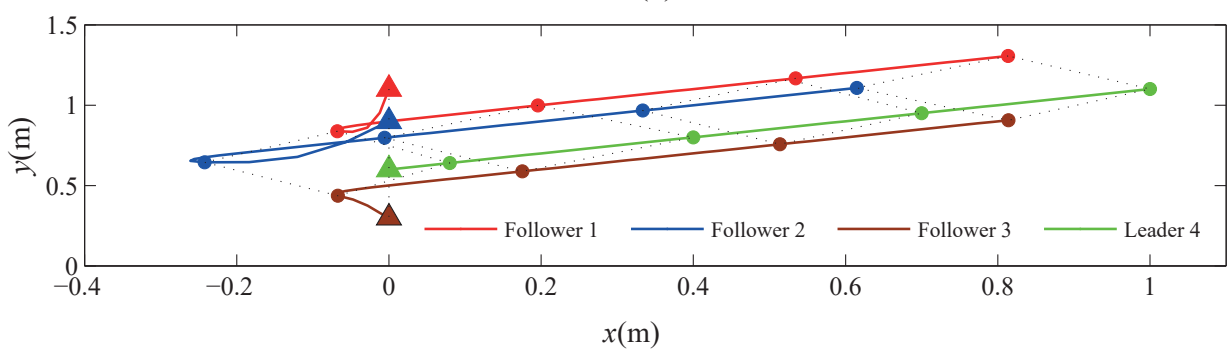

(b)

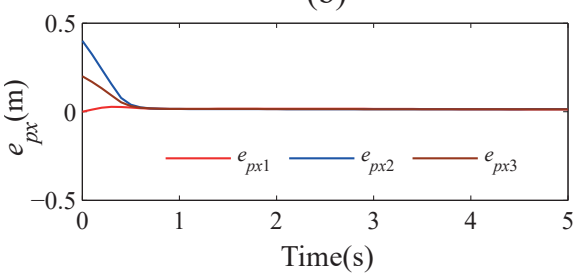

(e)

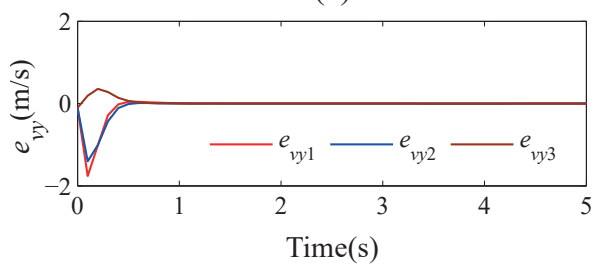

(c)

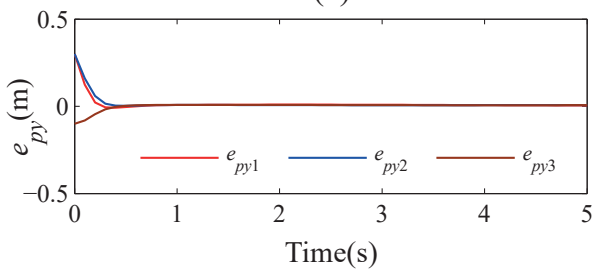

(f)

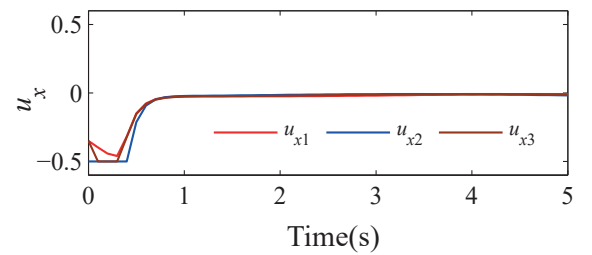

(d)

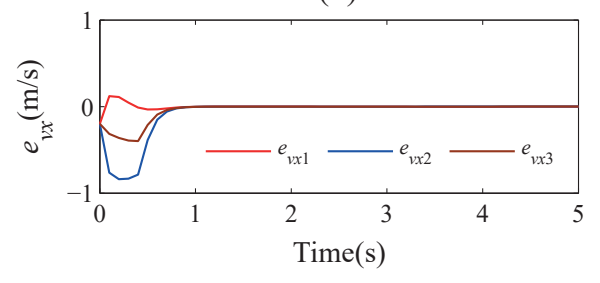

(g)

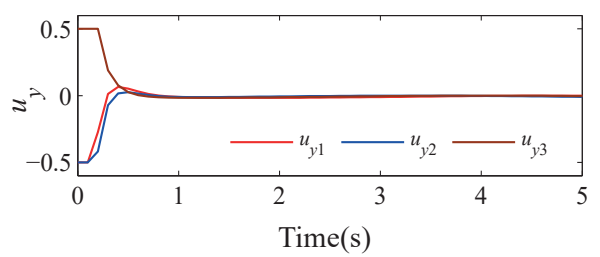

Fig. 4. Simulation results of the presented control scheme. (a) formation maneuvers in the Cartesian coordinate system, (b) curves of $e_{p x i}$ (c) curves of $e_{p y i},(\mathrm{~d})$ curves of $e_{v x i}$, (e) curves of $e_{v y i}$, (f) curves of $u_{x i}$, (g) curves of $u_{y i}(i=1,2,3)$

Consider the $i$ th follower agent $(i=1,2,3)$. Take its control design of the $x$-axis subsystem into account. Some parameters of the integral SMC-based controller should be predefined, that is, $c_{x i}=5$ and $\kappa_{x i}=1$. Concerning the NDO-based observer, its gain vector is chosen as $\mathbb{L}=\left[\begin{array}{ll}0 & 6\end{array}\right]^{T}$ by trial and error such that $\lambda_{x i}=\mathbb{L}^{T} \mathbb{B}=6$. The constant $\rho_{x i}$ in (17) is set by 1.1. The uncertain term of the $x$-axis subsystem is designed by $\delta_{x i}=0.02 \times \operatorname{rand}()$. Here $\operatorname{rand}()$ is a MATLAB command that generates a uniformly distributed random number in the closed interval $\left[\begin{array}{ll}-1 & 1\end{array}\right]$. Without loss of generality, the integral SMC-based controller and the NDO-based observer of the $y$-axis subsystem remain the same as corresponding parameters of the $x$-axis subsystem. Considering the motor load of these follower agents, both $u_{x i}$ and $u_{u i}$ are confined to $u_{x i} \leq 0.5$ and $u_{y i} \leq 0.5$.

Consider the following formation task. The leader agent moves along a straight line. The follower agents keep tracking the leader and form up into a diamond-shaped formation. The straight trajectory of the leader is presented as follows. In a Cartesian coordinate system, the initial coordinates of the leader are located at $[0 \mathrm{~m} 0.6 \mathrm{~m}]$. Correspondingly, its velocities in the $x$-direction and $y$-direction are set by $0.2 \mathrm{~m} \cdot \mathrm{s}^{-1}$ and $0.1 \mathrm{~m} \cdot \mathrm{s}^{-1}$, respectively. In order to form up into the desired diamond in this coordinate system, the initial coordinates of follower 1 , follower 2 and follower 3 in order are placed at $\left[\begin{array}{llll}0 & 1.1 & \mathrm{~m}\end{array}\right],\left[\begin{array}{lll}0 & \mathrm{~m} & 0.8\end{array}\right.$ $\mathrm{m}]$ and $[0 \mathrm{~m} 0.3 \mathrm{~m}]$, respectively. Their relative coordinations in order are assigned as $[-0.2 \mathrm{~m} 0.2 \mathrm{~m}],[-0.4 \mathrm{~m} 0 \mathrm{~m}]$ and $[-0.2 \mathrm{~m}-0.2 \mathrm{~m}]$ with regard to the leader agent.
Fig. 4 displays the simulation results of the presented control scheme by the multi-agent system. In Fig. 4a, the agents form up into the diamond-shaped formation from string one while moving in straight lines; filled triangles denote the initial positions of the agents and filled circles indicate the agents' positions in the dynamic process. In order to demonstrate the formation maneuver, the dash lines bond the agents together at the same moment. At the outset, the leader agent moves toward the right-half plane according to the designed trajectory. The three follower agents move towards the left-half plane in order to form up the desired diamond.

In Fig. 4b-e, the position errors and the velocity errors of each follower agent in the $x$ and $y$ directions are illustrated. According to the designed communication topology in Fig. 3, the position errors of follower 1 are defined by $e_{p x 1}=\left[x_{h 1}-\right.$ $\left.-\left(x_{h 2}-d_{12}^{x}\right)\right]+\left[x_{h 1}-\left(x_{h 4}-d_{14}^{x}\right)\right]$ and $e_{p y 1}=\left[y_{h 1}-\left(y_{h 2}-d_{12}^{y}\right)\right]+$ $+\left[y_{h 1}-\left(y_{h 4}-d_{14}^{y}\right)\right]$. Similarly, $e_{p x 2}=x_{h 2}-\left(x_{h 1}-d_{21}^{x}\right), e_{p y 2}=$ $=y_{h 2}-\left(y_{h 1}-d_{21}^{y}\right), e_{p x 3}=x_{h 3}-\left(x_{h 2}-d_{32}^{x}\right), e_{p y 3}=y_{h 3}-\left(y_{h 2}-\right.$ $\left.-d_{32}^{y}\right), e_{v x 1}=v_{x 1}-v_{x 2}+v_{x 1}-v_{x 4}, e_{v y 1}=v_{y 1}-v_{y 2}+v_{y 1}-v_{y 4}$, $e_{v x 2}=v_{x 2}-v_{x 1}, e_{v y 2}=v_{y 2}-v_{y 1}, e_{v x 3}=v_{x 3}-v_{x 2}$ and $e_{v y 3}=$ $=v_{y 3}-v_{y 2}$. From Fig. $4 \mathrm{~b}-\mathrm{e}$, these defined errors can converge to zero as the desired formation has been achieved. This indicates that the presented control scheme can realize the formation maneuver of the multi-agent system in spite of uncertainties. Further, the formation-control law of each follower agent is shown in Fig. 4f-g. 
(a)

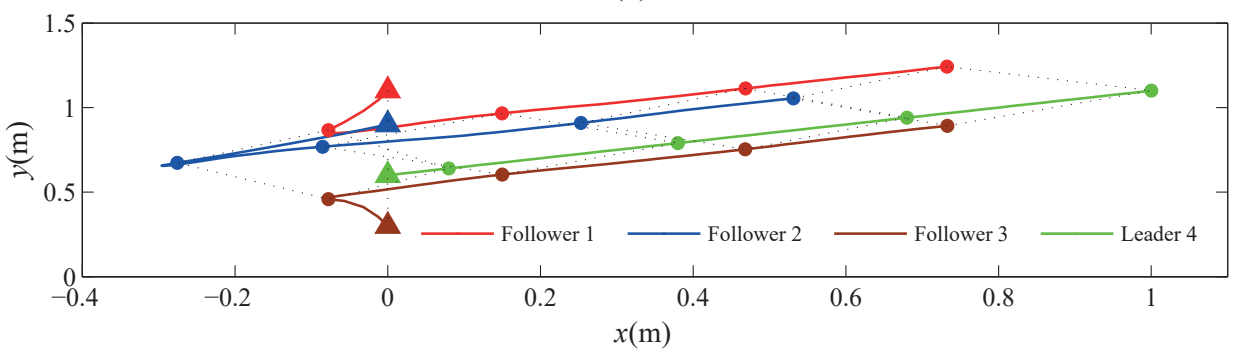

(b)

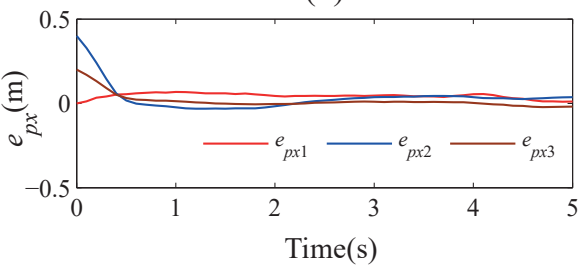

(e)

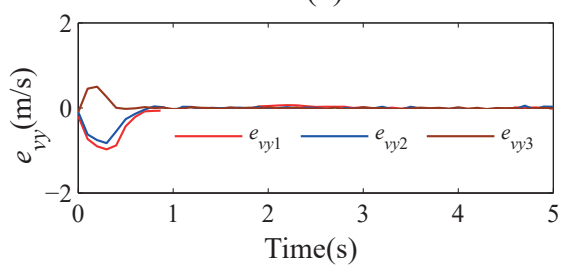

(c)

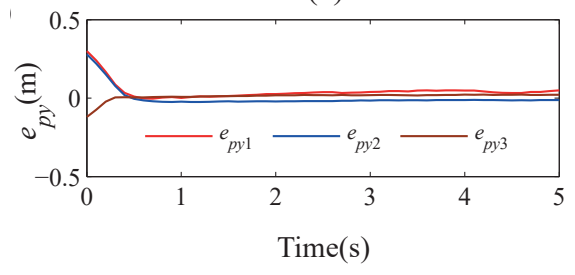

(f)

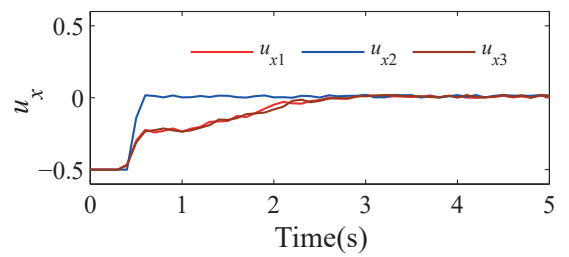

(d)

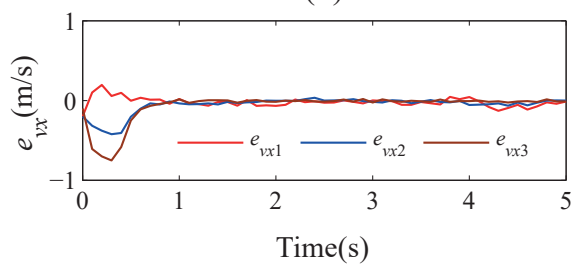

(g)

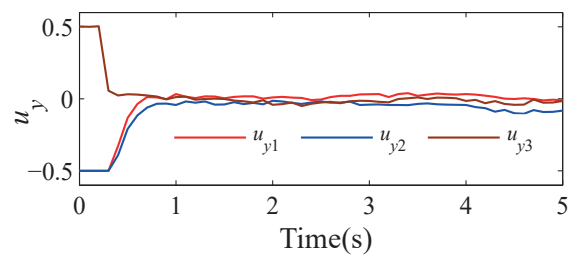

Fig. 5. Simulation results of the adaptive sliding mode control approach [16]: (a) formation maneuvers in the Cartesian coordinate system, (b) curves of $e_{p x i}$, (c) curves of $e_{p y i}$, (d) curves of $e_{v x i}$, (e) curves of $e_{v y i}$, (f) curves of $u_{x i}$, (g) curves of $u_{y i}(i=1,2,3)$

Fig. 5 illustrates the simulation results of the adaptive fuzzy sliding-mode control approach [16] by the same multi-agent system. These results in Fig. 5 are adopted for performance comparisons and our motivation is to highlight the superiority of the presented control scheme. As in Fig. 5a, the approach can also realize the same formation maneuver as the formation in Fig. 4a. However, our NDO-based integral sliding control method has better control performance in Fig. 4b-e via the comparisons in Fig. 5b-e.

Concerning the approach in [16], a fuzzy inference system (FIS) is designed to resist the uncertainties such that the control performance is subject to a number of fuzzy logic. The uncertainties in this paper are formulated by $0.02 \times \operatorname{rand}()$, compared with the expression of $0.005 \times \operatorname{rand}()$ in [16]. With the limited number of fuzzy rules, it is difficult for FIS to achieve better performance against the variations of uncertainties.

\section{Conclusions}

This paper has investigated the formation-control problem of multiple agents. The agents under consideration are wheeled mobile robots. The formation mechanism is leader-following. The uncertainties originated from each individual agent result in the formation uncertainties of the multi-agent system. It is mildly assumed that the formation uncertainties are bounded by an unknown boundary. In order to resist the formation un- certainties when forming up the agents, a control scheme that integrates the technique of NDO-based observer and the method of integral SMC is addressed. According to a given communication topology, the theoretical analysis proves that the formation control of the multi-gent system in the presence of uncertainties is of asymptotic stability. The control scheme has achieved the formation maneuvers by a multi-robot platform. The simulation results have demonstrated the effectiveness of the control scheme.

This paper has presented some theoretical results, as well as some numerical results for the multi-robot platform. In order to focus on the motivation of control design, some difficulties in application, such as communication delays and collisions between agents, are not considered during the control design. The no-communication-delay and no-collision conditions are mild enough for small-scale formations but they are rather idealized for large-scale formations. In order to take the presented control scheme into practical account, we continue to investigate this area and further works are in progress.

Acknowledgements. This work was partially supported by the National Natural Science Foundation of China Project under grants 60904008 and 61473176, the Natural Science Foundation of Shandong Province for Outstanding Young Talents in Provincial Universities (ZR2015JL021) and the International Science and Technology Cooperation Project between China and Denmark under grant No.2014DFG62530. 


\section{REFERENCES}

[1] A. Ratajczak, "Trajectory reproduction and trajectory tracking problem for the nonholonomic systems", Bull. Pol. Ac.: Tech. 64(1) 63-70 (2016)

[2] A. Byrski, R. Schaefer, M. Smolka and C. Cotta, "Asymptotic guarantee of success for multi-agent memetic systems", Bull. Pol. Ac.: Tech. 61(1), 257-278 (2013).

[3] L. Cheng, Y.Wang,W. Ren, Z.G. Hou and M. Tan, "On convergence rate of leader-following consensus of linear multi-agent systems with communication noises", IEEE Trans. Autom. Control 61(11), 3586-3592 (2016).

[4] Y. Kadowaki and H. Ishii, "Event-based distributed clock synchronization for wireless sensor networks", IEEE Trans. Autom. Control 60(8), 2266-2271 (2015).

[5] D. Pazderski and K. Kozłowski, "Control of a planar robot in the flight phase using transverse function approach", Bull. Pol. Ac.: Tech. 63(3) 759-770 (2015)

[6] Y. Dai, Y. Kim, S. Wee, D. Lee and S. Lee, "Symmetric caging formation for convex polygonal object transportation by multiple mobile robots based on fuzzy sliding mode control", ISA Trans. 60, 321-332 (2016).

[7] T.F. Liu and Z.P. Jiang, "Distributed nonlinear control of mobile autonomous multi-agents", Automatica, 50(4), 1075-1086 (2014).

[8] L. Cheng, Y. Wang, W. Ren, Z.G. Hou and M. Tan, "Containment control of multi-agent systems with dynamic leaders based on a PIn-type approach", IEEE Trans. Cybern, 46(12), 3004-3017, 2016.

[9] N.R. Esfahani and K. Khorasani, "A distributed model predictive control (MPC) fault reconfiguration strategy for formation flying satellites", Int. J. Control 89(5), 960-983 (2016).

[10] M. Salichon and K. Tumer, "Evolving a multiagent controller for micro aerial vehicles", IEEE Trans. Syst. Man Cybern. Part C-Appl. Rev. 42(6), 1772-1783 (2012).

[11] D.W. Qian, S.W. Tong, J.R. Guo and S. Lee, "Leader-followerbased formation control of nonholonomic mobile robots with mismatched uncertainties via integral sliding mode", Proc. Inst. Mech. Eng. Part I-J Syst Control Eng. 229(6), 559-569 (2015).

[12] D.W. Qian, S. Tong and C. Li, "Leader-following formation control of multiple robots with uncertainties through sliding mode and nonlinear disturbance observer", ETRI Journal 38(5), 1008-1018 (2016).

[13] W. Ren and R. Beard, Distributed Consensus in Multi-Vehicle Cooperative Controls. Springer-Verlag, London, 2007.

[14] Y. Dai, Y. Kim, S. Wee, D. Lee and S. Lee, "A switching formation strategy for obstacle avoidance of a multi-robot system based on robot priority model”, ISA Trans. 56, 123-134 (2015).

[15] Y. Chang, C. Chang, C. Chen and C. Tao, "Fuzzy sliding-mode formation control for multirobot systems: design and implementation”, IEEE Trans. Syst. Man Cybern. Part B-Cybern. 42(2), 444-457 (2012).

[16] Y. Chang, C. Yang, W. Chan, H. Lin and C. Chang, "Adaptive fuzzy sliding-mode formation controller design for multi-robot dynamic aystems", Int. J. Fuzzy Syst. 16(1), 121-131 (2014)

[17] M. Biglarbegian, "A novel robust leader-following control design for mobile robots”, J. Intell. Robot. Syst. 71, 391-402 (2013).
[18] H. Du, S. Li and X. Lin, "Finite-time formation control of multiagent systems via dynamic output feedback", Int. J. Robust Nonlinear Control 23(14), 1609-1628 (2013).

[19] B. Ranjbar-Sahraei, F. Shabaninia, A. Nemati and S. Stan, "A novel robust decentralized adaptive fuzzy control for swarm formation of multiagent systems" IEEE Trans. Ind. Electron. 59(8), 3124-3134 (2012).

[20] W. Zhang, J. Liu and H. Wang, "Ultra-fast formation control of high-order discrete-time multi-agent systems based on multistep predictive mechanism", ISA Trans. 58, 165-172 (2015).

[21] Y. Liu and Y. Jia, "Robust formation control of discrete-time multi-agent systems by iterative learning approach", Int. J. Syst. Sci. 46(4), 625-633 (2015).

[22] A. Zou and K. Kumar, "Neural network-based adaptive output feedback formation control for multi-agent systems", Nonlinear Dyn. 70(2), 1283-1296 (2012).

[23] K. Oh,M. Park and H. Ahn, "A survey of multi-agent formation control", Automatica 53, 424-440 (2015).

[24] C. Xu, Q. Zong, B.Tian and W. Xu, "Continuous sliding mode controller with disturbance observer for hypersonic vehicles", IEEE/CAA J Autom Sinica 2(1), 45-55 (2015).

[25] V. Gazi, B. Fidan, Y. Hanay and M. Köksal, "Aggregation, foraging, and formation control of swarms with non-holonomic agents using potential functions and sliding mode techniques", Turk. J. Electr. Eng. Comput. Sci. 15(2), 149-168 (2007).

[26] L. Dong, S. Chai, B. Zhang and S. Nguang, "Sliding mode control for multi-agent systems under a time-varying topology", Int. J. Syst. Sci. 47(9) 2193-2200 (2016).

[27] A. Zou, K. Kumar and Z. Hou, "Distributed consensus control for multi-agent systems using terminal sliding mode and Chebyshev neural networks", Int. J. Robust Nonlinear Control 23(3), 334-357 (2013).

[28] D. Zhao, T. Zou, S. Li and Q. Zhu, "Adaptive backstepping sliding mode control for leader-follower multi-agent systems", IET Contr. Theory Appl. 6(8), 1109-1117 (2012).

[29] D. Qian, S. Tong, H. Liu and X. Liu, "Load frequency control by neural-network-based integral sliding mode for nonlinear power systems with wind turbines", Neurocomputing 173, 875-885 (2016).

[30] H. Sun, S. Li and C. Sun, "Robust adaptive integral-slidingmode fault-tolerant control for airbreathing hypersonic vehicles", Proc. Inst. Mech. Eng. Part I-J Syst Control Eng. 226(10), 1344-1355 (2012).

[31] Y. Jiang, J. Liu and S. Wang, "Robust integral sliding-mode consensus tracking for multi-agent systems with time-varying delay", Asian J. Control 18(1) 224-235 (2016).

[32] S. Yu and X. Long, "Finite-time consensus for second-order multi-agent systems with disturbances by integral sliding mode", Automatica 54, 158-165 (2015).

[33] W. Chen, J. Yang, L. Guo and S. Li, "Disturbance-observerbased control and related methods-an overview", IEEE Trans. Ind. Electron. 63(2), 1083-1095 (2016).

[34] M. Kim and W. Chung, "Disturbance-observer-based PD control of flexible joint robots for asymptotic convergence", IEEE Trans. Robot. 31(6), 1508-1516 (2015).

[35] K.D. Do and J. Pan, Control of Ships and Underwater Vehicles, Springer-Verlag, Berlin, 2009. 